Original Article

\title{
Effects of a Fall Prevention Exercise Program on Muscle Strength and Balance of the Old-old Elderly
}

\author{
SeOnG-IL $\mathrm{CHO}^{1)}$, DuK-Hyun $\left.\mathrm{An}^{2}\right)^{*}$ \\ 1) Department of Rehabilitation Science, Graduate School, Institute for Safety Promotion, Inje \\ University, Republic of Korea \\ 2) Department of Physical Therapy, College of Biomedical Science and Engineering, Inje University: \\ 607 Obang dong, Gimhae, Gyeongsangnam-do 621-749, Republic of Korea
}

\begin{abstract}
Purpose] The purpose of this study was to investigate the effects of an 8-week balance exercise and elastic-resistance exercise program on muscle strength and balance of the old-old elderly (over the age of 75). [Subjects and Methods] Fifty-five elderly persons were recruited from the community and assigned to three groups for convenience: balance exercise (intervention group 1; INT 1), resistance exercise (intervention group 2; INT 2), and control (CON) groups. The intervention was performed twice a week at a senior center and three times a week at home for 8 weeks. Muscle strength and balance were evaluated before and at the end of the trial, using a PowertrackIIand Tetrax. [Results] There were significant improvements in the strength of all seven muscle groups and balance in the INT 2 group. In the INT 1 group, there were significant improvements in the strength of all muscle groups except for the knee flexor and ankle plantar flexor muscle groups. [Conclusion] This study demonstrated that an intervention using balance exercises or elastic-resistance exercises is effective at improving the muscle strength and balance of the old-old elderly. These type of exercises should be appropriate for the physical characteristics of the subjects

Key words: Falls, Muscle strength, Postural balance
\end{abstract}

(This article was submitted Apr. 10, 2014, and was accepted May 16, 2014)

\section{INTRODUCTION}

The elderly have at least a 10 times higher risk of falling than other age groups because of age-related physiological changes, especially those pertaining to the quality and quantity of skeletal muscles ${ }^{1)}$. Thirty to fifty percent of the elderly over 65 years of age experience falls every year ${ }^{2)}$. The physical damage from falls may include bruising, hematoma, fractures, and even brain damage or other secondary complications, and decreasing physiological functions and weakening muscles may lead to other falls ${ }^{3)}$. Although falls may not necessarily lead to extreme physical damage, they may restrict daily activities because of long-term pain $^{4}$. For the elderly, the high-risk factors of falls are loss of muscle strength and balance instability ${ }^{5,6)}$. Therefore, fall-prevention exercises for the elderly consist of lower extremity resistance exercises and balance training ${ }^{7}$.

There is currently a need for a specialized fall-prevention program that considers the special characteristics of elderly persons in their $80 \mathrm{~s}$, because existing programs are not effective enough for this high-risk group ${ }^{8)}$. Newman

*Corresponding author. Duk-Hyun An (E-mail: dhahn670208@gmail.com)

(C2014 The Society of Physical Therapy Science. Published by IPEC Inc. This is an open-access article distributed under the terms of the Creative Commons Attribution Non-Commercial No Derivatives (by-ncnd) License $<$ http://creativecommons.org/licenses/by-nc-nd/3.0/> . and Newman ${ }^{9)}$ argued that fall-prevention programs focusing on physical abilities are not highly effective because the elderly over the age of 75 show a rapid decrease in instrumental activities of daily living compared with elderly persons between aged 60 to 74 years of age. After measuring and comparing the strength differences among different age groups, Lamoureux et al. ${ }^{10)}$ reported that people's strength weakens as they age. This occurs rapidly after the age of 75 , when serious muscle weakening in the lower extremities begins. Rogers et al. ${ }^{11)}$ suggested that the frequency of falls actually increases over the age of 75 . However, the old-old elderly (over the age of 75) have little opportunity to participate in regular physical activities, which makes it harder for them to increase their physical activity and strength ${ }^{12)}$. As people age, there is a higher chance of death because of complications and falls and less chance of recovery ${ }^{13)}$. Thus, an intervention for fall prevention is needed to increase the balance and strength of the old-old elderly.

Purpose of this study was to investigate the effects of balance exercise and elastic-resistance exercise programs on the muscle strength and balance of the old-old elderly in order to suggest an effective intervention for fall prevention for this population, who are at the highest risk of falls.

\section{SUBJECTS AND METHODS}

\section{Subjects}

Sixty elderly persons over the age of 75 who lived in the community were chosen by convenience sampling as par- 
ticipants for this study. They gave their written informed consent to participation in the study after the experimental procedures had been explained. The study was approved by the Inje University Faculty of Health Science Human Ethics Committee. Subjects were excluded if they had neurological impairments, severe cardiovascular diseases, persistent joint pain, or musculoskeletal impairment; required assistance from another person or a device during ambulation; scored lower than 24 on the Mini-Mental State Examination (Korean version); or were on medication that affected balance. Subjects who were already involved in other exercise programs were also excluded from this study.

To prevent the diffusion of the experiment's intervention, three senior citizen centers that were far from one another were chosen, and 20 participants were chosen from each center. Four participants dropped out from the intervention group 1 (INT 1 group), one dropped out from the intervention group 2 (INT 2 group), and none dropped out from the control (CON) group. Therefore, the final number of the participants was 55. Their general characteristics are shown in Table 1.

\section{Methods}

Strength of the hip flexors, hip extensors, hip abductors, knee flexors, knee extensors, ankle dorsiflexors, and ankle plantar flexors was measured using a hand-held dynamometer (Powertrack II, JTECH Medical). Strength was measured directly by the experimenter to prevent intertester error. The participants were explained the procedure of each measurement and performed one trial for each. Then, three measurements were taken and the average value was calculated. To find the interrater reliability for each muscle, the intraclass correlation coefficient (ICC) of each muscle strength measurement was calculated, and the values ranged from 0.97 to 0.99 . The fall index, which evaluates the risk of falls, was calculated using a Tetrax (Sunlight Medical Ltd., Israel) to measure balance. The balance index ranges from 0 to 100 , with a higher score indicating a higher risk of falls.

The INT 1 group performed balance exercises and the INT 2 group performed elastic-resistance exercises in 1-hour sessions five times a week for 8 weeks, a total of 40 sessions. The experimenters conducted the group exercises two times a week at the senior citizen centers, and the individuals performed the exercises at home, by themselves, three times a week. All 1-hour sessions consisted of 10 minutes of warm-up and stretching, 40 minutes of main exercise, and 10 minutes of cool down. The exercises were conducted in 3 sets, with 10 repetitions per set, and there was a 30 -second rest period between each set and a 3- to 5-minute rest period between each exercise. A safety device was employed to prevent falls during the exercises. The CON group performed the exercises after the 8-week intervention period had ended.

The balance exercises consisted of six types of exercise: side stepping, tandem walking, retro walking, braiding, one-leg stance with cup tapping, and external perturbation while standing with the head up and back straight. The elastic-resistance exercises consisted of eight types of exercise: squats, heel raise, hip flexion/extension, knee flexion/exten-
Table 1. General characteristics of the subjects $(\mathrm{N}=55)$

\begin{tabular}{lrrr}
\hline Variable & INT 1 $(\mathrm{n}=16)$ & INT 2 $(\mathrm{n}=19)$ & $\mathrm{CON}(\mathrm{n}=20)$ \\
\hline Age (years) & $76.1(4.2)^{*}$ & $78.1(3.7)$ & $75.9(1.8)$ \\
Weight $(\mathrm{kg})$ & $59.6(3.6)$ & $55.8(8.4)$ & $60.2(7.5)$ \\
Height $(\mathrm{cm})$ & $156.5(3.6)$ & $159.5(6.7)$ & $154.5(6.3)$ \\
\hline
\end{tabular}

*mean (SD)

INT $1=$ intervention group 1 , INT $2=$ intervention group 2, $\mathrm{CON}=$ control group

sion, and ankle plantar/dorsiflexion. Resistance was provided by Thera-Bands (Hygenic Corporation, Akron, $\mathrm{OH}$, USA) and the intensity and frequency of the exercises were chosen following to previous studies (e.g., Zion et al. ${ }^{12)}$ ). Red and yellow elastic bands were chosen at the appropriate level for the seniors. The level of the bands was gradually raised, and it was also raised during the last set when a participant was able to perform the actions without much difficulty. The exercises were conducted slowly so that concentric and eccentric contraction would occur accurately.

The collected data were statistically processed using SPSS version 18.0 for Windows (SPSS Inc., Chicago, IL, USA). Analysis of variance (ANOVA) was used as a homogeneity test of the muscle strengths and balance abilities of the INT 1, INT 2, and CON groups. The paired t-test was used to examine the differences between the pretest and posttest values of balance and muscle strength to determine the program's effectiveness. Also, ANOVA was used to assess the significance of differences among the populations of the INT 1, INT 2, and CON groups. Bonferroni adjusted post hoc tests were conducted to identify the comparisons that were statistically significant. Significance was accepted for values of $p<0.01$. The ICC was calculated to test the reliability of the equipment.

\section{RESULTS}

The homogeneity test for the muscle strength and fall index values of the INT 1, INT 2, and CON groups before the intervention found no significant differences among the three groups. The INT 1 group showed statistically significant improvements in muscle strength in five muscle groups: hip flexor $(\mathrm{t}=-5.21, \mathrm{p}<0.001)$, hip extensor $(\mathrm{t}=$ $-3.57, \mathrm{p}=0.003)$, hip abductor $(\mathrm{t}=-6.52, \mathrm{p}<0.001)$, knee extensor $(\mathrm{t}=-6.79, \mathrm{p}<0.001)$, and ankle dorsiflexor $(\mathrm{t}=$ $-4.28, \mathrm{p}=0.001)$. The strengths of the knee flexor $(\mathrm{t}=-1.78$, $\mathrm{p}=0.096)$ and ankle plantar flexor $(\mathrm{t}=-1.67, \mathrm{p}=0.115)$ improved after the intervention, but without significant difference. The INT 2 group showed statistically significant improvements in the strengths of all the muscle groups tested: hip flexor $(\mathrm{t}=-5.12, \mathrm{p}<0.001)$, hip extensor $(\mathrm{t}=-3.55$, $\mathrm{p}=0.002)$, hip abductor $(\mathrm{t}=-5.11, \mathrm{p}<0.001)$, knee flexor $(\mathrm{t}=-3.67, \mathrm{p}=0.002)$, knee extensor $(\mathrm{t}=-12.27, \mathrm{p}<0.001)$, ankle dorsiflexor $(\mathrm{t}=-7.93, \mathrm{p}<0.001)$, and ankle plantar flexor $(\mathrm{t}=-6.05, \mathrm{p}<0.001)$. After the intervention, the fall index score of the INT 1 group decreased from 59.0 (22.0) to 44.5 (17.4), a statistically significant decrease $(t=5.77, \mathrm{p}$ $<0.001)$. The INT 2 group also showed a statistically significant decrease in the fall index score from 62.5 (24.9) to 
41.9 (23.7). However, the CON group showed no significant differences in the strengths of any muscle group or in the fall index after 8 weeks.

ANOVA of the differences between the pretest and posttest mean values of the INT 1, INT 2, and CON groups found significant differences for all seven muscle groups and balance ability among the three groups. The post hoc test, further revealed that the INT 1 group showed statistically significant improvements in the hip flexor, hip extensor, hip abductor, knee extensor, and ankle dorsiflexor muscles, and the fall index compared to the pre-post mean difference of the CON group. The INT 2 group showed statistically significant improvements in all seven types of muscles and the fall index compared to the CON group. The INT 2 group showed a significant mean difference in the ankle plantar flexor muscle compared to the INT 1 group.

\section{DISCUSSION}

The purpose of this study was to investigate the effects of balance exercise and elastic-resistance exercise programs on the muscle strength and balance of the old-old elderly. The INT 1 group showed a statistically significant improvement in muscle strengths of five of seven muscle groups, but not those of the knee flexors and ankle plantar flexors. Compared to the mean muscle strengths of the $\mathrm{CON}$ group, after the balance exercises, the INT 1 group showed statistically significant improvements in the strengths of the five muscle groups. After conducting balance exercises for seniors over the age of 65 , Kim and Lockhart ${ }^{14)}$ reported an improvement in muscle strength and gait and concluded that balance exercises contributes to fall prevention.

The INT 2 group showed a statistically significant improvement in the strength of all seven muscle groups after the intervention. In the comparison of mean muscle strengths with those of the CON group, after the resistance exercises, the INT 2 group showed significant improvements in the strengths of all seven muscle groups. This result is in accordance with the results of Zion et al. ${ }^{12)}$, who conducted elastic-resistance exercises over the course of 8 weeks for the elderly and found that muscle strength and balance showed statistically significant improvements. Kim and Lockhart ${ }^{14)}$ reported that the ankle plantar flexor and knee extensor strengths of 18 older adults improved after performance of elastic-resistance exercises for 8 weeks. As people age, the risk of fall increases because a decrease muscle mass leads to atrophy, which decreases muscle function ${ }^{15)}$. Strengthening exercises using elastic resistance are practical because they have only a small risk of injury, provide consistent tension, and are easily portable and inexpensive. Elastic-resistance exercises help not only in rehabilitation but also in other areas because they have been proven to improve muscle strength and stability ${ }^{16)}$.

The INT 1 and INT 2 groups showed significant improvements in balance after the intervention, which proves that both balance exercises and resistance exercises are effective at improving balance. In the posttest comparison of the mean differences in balance among the three groups, no difference was found between the INT 1 and INT 2 groups, but both groups showed significant improvements in comparison with the CON group. Age-related balance deficits may occur because of increased postural sway caused by weakness of leg muscles and decreased proprioception ${ }^{17)}$. The balance exercises and elastic-resistance exercises conducted for 8 weeks improved leg muscle strength and proprioception of the neuromuscular system through muscle contraction and stimulation of proprioceptive sense. Lee and Park ${ }^{18)}$ reported that improvement in the strength of the lower extremities enhances the balance of the elderly. We assume that the improvement in balance observed in the present study resulted from the improvement seen in the strength of the lower limbs.

Unlike previous studies that have reported fall-prevention exercises to be ineffective for the old-old elderly ${ }^{8,9)}$, our results show that balance exercises and elastic-resistance exercises were effective at improving the muscle strength and balance of this population. Skelton and Beyer ${ }^{19)}$ indicated that there are limits to the effectiveness of fall-prevention exercises for the old-old elderly, and noted that one-onone approaches for the elderly are more effective than group exercises. In our study, the intervention was conducted for the old-old elderly, who have a rapid aging process, and thus are the most vulnerable to falls. Our results show there were improvements in both the balance exercise and resistance exercise groups after 8 weeks. Although the elasticresistance exercises proved to be more effective than the balance exercises at strengthening the ankle plantar flexors, there was not a big difference in the effectiveness of the two exercise types; thus, we conclude that both types of exercise are acceptable as fall-prevention exercises for the old-old elderly. We also suggest that these exercises be used appropriately according to personal physical characteristics and needs. Because of the physical characteristics of the elderly, fall-prevention exercises should not pose injury risks, and should be easy to practice, even at home. The balance exercises and elastic-resistance exercises used in the present study are accessible to the elderly, because they do not need specialized equipment and can be adjusted to personal physical strength.

A limitation of this study was that the group exercises with the researcher were conducted for only 2 of the 5 intervention days per week, leaving 3 days per week for the elderly to perform the exercises by themselves. This made it difficult to control the intervention perfectly. The significance of this study is that it revealed that balance exercises and elastic-resistance exercises are effective at fall prevention for the old-old elderly, who are at high risk of falls because of rapid aging. Therefore, such exercises are suggested for fall prevention programs for this particular population.

\section{REFERENCES}

1) Miller CA: The connection between drugs and falls in elders. Geriatr Nurs, 2002, 23: 109-110. [Medline] [CrossRef]

2) Menz HB, Lord SR, Fitzpatrick RC: A structural equation model relating impaired sensorimotor function, fear of falling and gait patterns in older people. Gait Posture, 2007, 25: 243-249. [Medline] [CrossRef]

3) Jung Y, Lee S, Chung K: Prevalence and associated factors of falls according to health status in elderly living in the community. J Korean Gerontol, 
2006, 26: 291-303.

4) Hartholt KA, van Beeck EF, Polinder S, et al.: Societal consequences of falls in the older population: injuries, healthcare costs, and long-term re duced quality of life. J Trauma, 2011, 71: 748-753. [Medline] [CrossRef]

5) Rubenstein LZ: Falls in older people: epidemiology, risk factors and strategies for prevention. Age Ageing, 2006, 35: ii37-ii41. [Medline] [CrossRef]

6) Rubenstein LZ, Josephson KR: The epidemiology of falls and syncope Clin Geriatr Med, 2002, 18: 141-158. [Medline] [CrossRef]

7) Granacher U, Muehlbauer T, Zahner L, et al.: Comparison of traditiona and recent approaches in the promotion of balance and strength in older adults. Sports Med, 2011, 41: 377-400. [Medline] [CrossRef]

8) Park S: Meta-analysis of the interventions for preventing falls by the elderly in the eight countries: comparison between aged 70's and 80 's. J Korean Gerontol, 2010, 30: 49-63.

9) Newman BM, Newman PR: Development Through Life: A Psychosocial Approach, 10th ed. Belmont: Wadsworth, 2009, pp 533-565.

10) Lamoureux EL, Sparrow WA, Murphy A, et al.: Differences in the neuromuscular capacity and lean muscle tissue in old and older communitydwelling adults. J Gerontol A Biol Sci Med Sci, 2001, 56: M381-M385. [Medline] [CrossRef]

11) Rogers ME, Rogers NL, Takeshima N, et al.: Methods to assess and improve the physical parameters associated with fall risk in older adults. Prev Med, 2003, 36: 255-264. [Medline] [CrossRef]
12) Zion AS, De Meersman R, Diamond BE, et al: A home-based resistancetraining program using elastic bands for elderly patients with orthostatic hypotension. Clin Auton Res, 2003, 13: 286-292. [Medline] [CrossRef]

13) Guideline for the prevention of falls in older persons. American Geriatrics Society, British Geriatrics Society, and American Academy of Orthopaedic Surgeons Panel on Falls Prevention. J Am Geriatr Soc, 2001, 49: 664-672. [Medline]

14) Kim S, Lockhart $\mathrm{T}$ : Effects of 8 weeks of balance or weight training for the independently living elderly on the outcomes of induced slips. Int J Rehabil Res, 2010, 33: 49-55. [Medline] [CrossRef]

15) Barry BK, Carson RG: The consequences of resistance training for movement control in older adults. J Gerontol A Biol Sci Med Sci, 2004, 59: 730-754. [Medline] [CrossRef]

16) Patterson RM, Stegink Jansen CW, Hogan HA, et al.: Material properties of Thera-band tubing. Phys Ther, 2001, 81: 1437-1445. [Medline]

17) Edelberg HK: Falls and function. How to prevent falls and injuries in patients with impaired mobility. Geriatrics, 2001, 56: 41-45, quiz 49. [Medline]

18) Lee IH, Park SY: Balance improvement by strength training for the elderly. J Phys Ther Sci, 2013, 25: 1591-1593. [Medline] [CrossRef]

19) Skelton DA, Beyer N: Exercise and injury prevention in older people Scand J Med Sci Sports, 2003, 13: 77-85. [Medline] [CrossRef] 